\title{
Tissue necrosis following extravasation of acyclovir in an adolescent: A case report
}

\author{
Charalambos Neocleous, Eleni Andonopoulou, Alkistis Adramerina, \\ Antigoni Pegkou, Olga Savelieva, Petroula Georgiadou, Ioannis Drikos
}

\author{
Department of Medical Pediatrics \\ Thriasio General Hospital, Athens \\ Greece \\ Correspondence: \\ xneoc@yahoo.gr \\ Tel.: + 306955099935 \\ Fax.: + 302105546039
}

Received: 12 February 2017

Accepted: 2 May 2017

Key words: Tissue necrosis Extravasation - Acyclovir.

\begin{abstract}
Objective. Extravasation of intravenously infused vesicant solutions is a common problem in medical practice, which can lead to severe and progressive tissue dysfunction, ranging from persistent tissue oedema and fibrosis to delayed tissue necrosis. Acyclovir is a known vesicant medication administrated in paediatric patients, which appears to irritate venous and soft tissue if extravasated. Case report. We present the first case involving the extravasation of intravenously infused acyclovir in a female adolescent patient, which caused tissue necrosis and left behind a residual scar lesion. Nursing and medical staff should be aware of the potential dermatological side effects of intravenously infused acyclovir and other medications, even a long time after infusion, and the possible lack of initial local symptoms and signs. Conclusion. Early recognition of extravasation and prompt management are critical in preventing further morbidity, and optimizing outcomes.
\end{abstract}

\section{Introduction}

Extravasation of intravenously infused vesicant solutions is a common problem in medical practice, which can lead to severe and progressive tissue dysfunction, ranging from persistent tissue oedema and fibrosis to delayed tissue necrosis $(1,2)$. Acyclovir is a known vesicant medication administrated in paediatric patients, which appears to irritate venous and soft tissue if extravasated $(3,4)$. We report here the first case involving extravasation of intravenous (IV) acyclovir in a female adolescent patient, which caused tissue necrosis and left behind a residual scar lesion.

\section{Case presentation}

A 14-year-old female patient was admitted to our hospital for a two-day-long fever and headache. On admission, her general condition was affected and the patient appeared drowsy. Her temperature reached $37.9^{\circ} \mathrm{C}$. A neurological examination revealed neck rigidity with negative Kerning and Brudzinski signs, while a further physical examination revealed no remarkable findings. A lumbar puncture revealed clear cerebrospinal fluid with 15 leucocytes $/ \mathrm{mm}^{3}$ (40\% polymorphs and $60 \%$ lymphocytes). The levels of glucose and protein in the cerebral vascular fluid were within normal limits. A cerebrospinal fluid (CSF) specimen was cultured for common bacterial pathogens of the central nervous system (CNS), and it was also examined by PCR for common bacterial and viral pathogens (including Herpes Simplex Virus (HSV1 and HSV2) of CNS.

Haemoglobin, total leukocyte count, Creactive protein, ESR, serum electrolytes, 
and renal and liver function tests were within normal limits. Due to the initial altered mental status, fever and increased CSF leucocyte count, HSV meningoencephalitis was considered as possible; as such, empirical therapy with IV acyclovir was started. Acyclovir $(30 \mathrm{mg} / \mathrm{kg} / 24 \mathrm{~h}$ in three divided doses) was administered in $100 \mathrm{~mL}$ of $0.9 \%$ sodium chloride as hourly IV infusions.

Venous access was placed on the right forearm. Before infusion, the area around the catheter was routinely checked for inflammation or signs of infection. On the fifth day, 10 min after acyclovir administration, the patient complained of mild burning at the catheter insertion site. The IV line was checked and, due to the lack of patency, the infusion was interrupted. It was unknown how long the IV line had not been patent. However, the patient showed no complaints or local symptoms, which suggested inflammation until the moment that a mild sensation of burning was indicated. A painless $0.5 \times 0.5 \mathrm{~cm}$ large bullous lesion was observed, with regular boundaries in the area around the catheter and along the tract of the vein. The bulla was not surrounded by erythema or induration.

The bullous lesion was opened and serous fluid was drained by plastic surgeons. An examination of the underlying tissue revealed skin breakdown and necrosis (dark black colour) (Figure 1). We opted for non-surgical treatment. The patient underwent $72 \mathrm{~h}$ of close observation with compartment checks, topical antiseptic and antibiotic treatment. Despite the fact that both the PCR results in CFS and the results of the cultures were negative, the therapy with acyclovir was continued because the HSV PCR test can result in false negatives, particularly among children, and early on in the course of disease (5). For the remaining nine days of treatment, IV acyclovir was infused through venous access on the other hand. After discharge from the hospital, the patient returned for a follow-up one month later. Initial evaluation revealed a residual scar at the area of the initial skin lesion, although the patient was completely asymptomatic. At the patient's request, a scar revision for aesthetic reasons was scheduled.

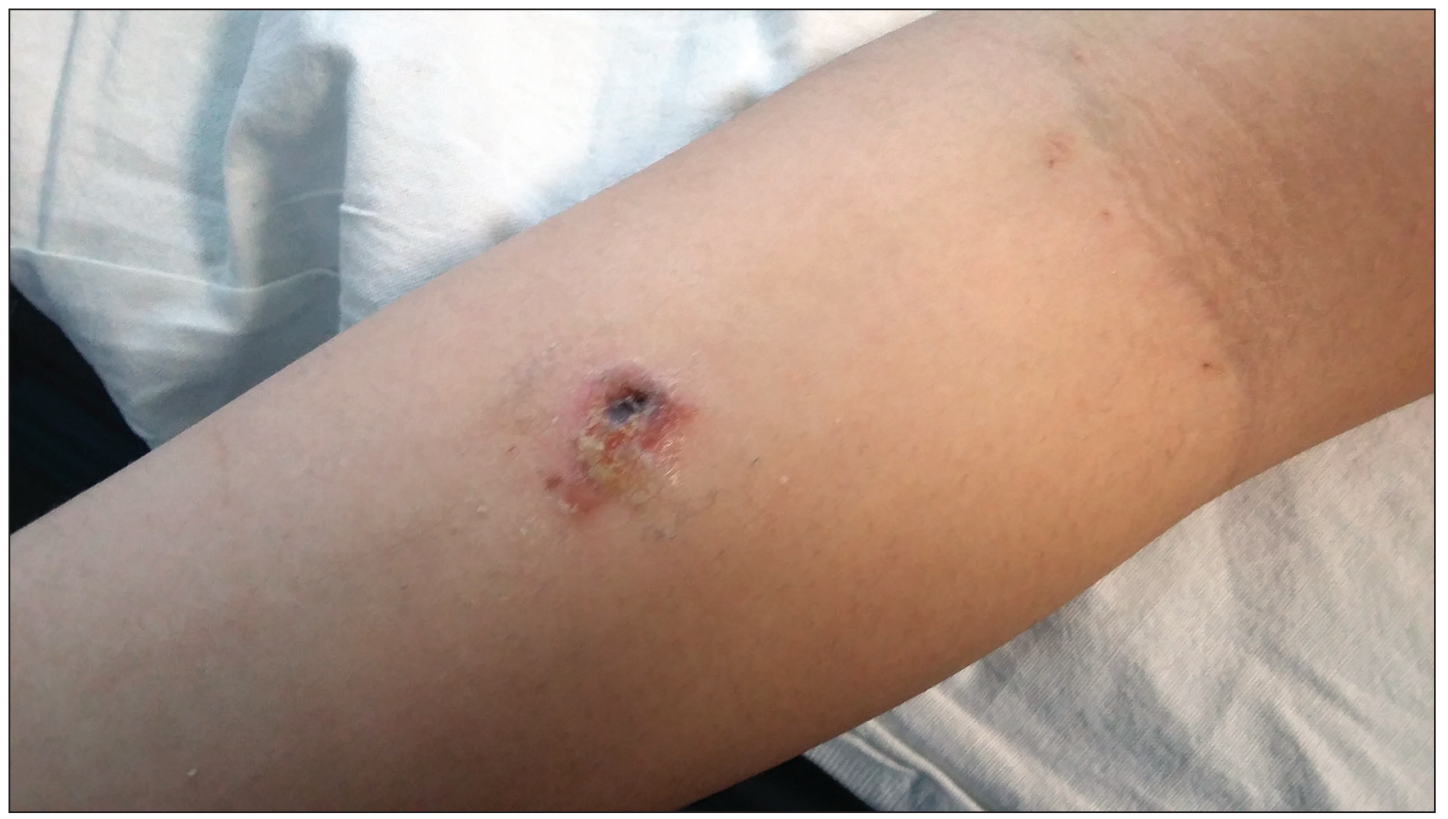

Figure 1 Tissue necrosis following extravasation of acyclovir. 


\section{Discussion}

The reported dermatological adverse effects of acyclovir, including erythema, inflammation and phlebitis at the injection site, occur in $\leq 16 \%$ of patients (6). The solution is highly alkaline ( $\mathrm{pH}$ of 11 , osmolality of 278 $\mathrm{mOsm} / \mathrm{kg}$ ), which means that extravasation may cause significant tissue damage (4).

In the literature, only three cases of extravasation of IV acyclovir have been reported so far $(1,3,4)$. De Souza and Shibu reported a 51-year-old insulin-dependent diabetic man, who presented with cellulitis and lymphatic oedema over the prior site of extravasation three months after infusion. Meanwhile, Sarica reported the case of a 14-year-old girl with acute lymphoblastic leukaemia who was given IV acyclovir for chicken pox. On the 33rd day of remission induction, the infusion extravasated and a local bullous cutaneous eruption occurred approximately $10 \mathrm{~cm}$ distal to the site of venepuncture. The bullous eruption subsided in eight hours and completely resolved, with a residual scar, in 24 hours. Lau and Lee reported on a 55-year-old male with suspected herpes simplex encephalitis and known HIV who was treated with IV acyclovir. A diffuse swelling, which was firm but compressible, of the right hand was noticed on the second day in hospital following extravasation of $150 \mathrm{~mL}$ of IV acyclovir (1). In all case reports, treatment was non-operative with compression garments and physical therapy (4), cold compresses and arm elevation in a skyhook (1), or observation (3). Additionally, in previous cases, no apparent skin breakdown or necrosis was noted.

Our report presents the first case of IV acyclovir extravasation in an immunocompetent female adolescent, which caused tissue necrosis and left a residual scar. Similar to the patients discussed above, we believe that tissue necrosis occurred in our case because of the alkalinity of the extravasated solution, resulting in local chemical inflammation and tissue damage. However, extravasation of IV medications usually presents as local swelling, erythema and pain. Only in severe cases or in patients presenting or referring late in time has serious injury including tissue necrosis and compartment syndrome been observed (4).

In contrast to previous patients, our patient was diagnosed with tissue necrosis without presenting any other symptom (pain or burning) or findings (swelling) early on in the course of tissue damage. The patient only complained about mild burning at the time when tissue necrosis was diagnosed. We believe that the lack of such symptoms and findings early on in the course of tissue damage was the main reason for the delay in diagnosis and subsequent severe tissue damage. De Souza and Shibu suggested that diabetic neuropathy was the reason why their patient did not experience any pain connected with the extravasation injury. In our patient, there was no obvious reason to explain the lack of symptoms related to the extravasation injury.

We urge medical staff to consider the potential dermatological side effects of vesicant medications, which include blistering, sloughing of the skin and varying degrees of localized tissue damage when they inadvertently leak into the tissue. Extravasation of acyclovir may also cause late complications, such as persistent lymphatic oedema, cellulitis, tissue fibrosis and necrosis. Extravasation of vesicant medications is thus a medical emergency.

\section{Conclusion}

Extravasation of vesicant medication, a common incident in medical practice, can cause severe tissue dysfunction. Nursing and medical staff should consider the potential dermatological side effects of IV acyclovir (even a long time after infusion) and 
the possible lack of initial local symptoms after the extravasation. Early recognition of extravasation and prompt management are critical in preventing further morbidity and optimizing outcomes.

\section{What is already known on this topic}

Acyclovir is a known vesicant medication administrated in paediatric patients, which appears to irritate venous and soft tissue if extravasated. However, only three patients with extravasation of IV acyclovir have been reported so far. In these case reports, no apparent skin breakdown or necrosis was not$e d$, while the treatment was non-operative.

\section{What this study adds}

We present the first patient with extravasation of IV acyclovir, which caused tissue necrosis and left behind a residual scar. In contrast to the previously reported patients, our patient was diagnosed with tissue necrosis without any other symptoms or findings early on in the course of tissue damage. It is thus important to remain vigilant at the time of the IV administration of vesicant medications in children. Early recognition of extravasation and prompt management could prevent further morbidity and optimize outcomes.

Authors' contributions: Conception and design: $\mathrm{CN}$ and EA; Acquisition, analysis and interpretation of data: $\mathrm{CN}, \mathrm{ID}$, OS and AA; Drafting the article: $\mathrm{CN}$,
OS, AP and PG; Revising it critically for important intellectual content: CN, ID, EA and AA; Approved final version of the manuscript: $\mathrm{CN}$, ID and AA.

Conflicts of interest: The authors declare that they have no conflict of interest.

\section{References}

1. Lau BC, Lee NH. Acyclovir Extravasation: Case Report and Review of the Literature. Austin J Orthopade \& Rheumatol. 2016;3(1):1026.

2. Kumar RJ, Pegg SP, Kimble RM. Management of extravasation injuries. ANZ J Surg. 2001;71(5):285-9.

3. Sarica A. Bullous Cutaneous Eruption due to Extravasation of Acyclovir in an Adolescent with Acute Lymphoblastic Leukemia. Turk J Haematol. 2012;29(1):109-10.

4. De Souza BA, Shibu M. Painless acyclovir extravasation injury in a diabetic. Br J Plast Surg. 2002;55(3):264.

5. Arun V, Romergryko GG. Diagnosis and management of acute encephalitis. A practical approach. Neurol Clin Pract. 2014;4(3):206-15.

6. Buck ML, Vittone SB, Zaglul HF. Vesicular eruptions following acyclovir administration. Ann Pharmacother. 1993;27(12):1458-9. 